\title{
Genome Sequence and Comparative Analysis of Colletotrichum gloeosporioides Isolated from Liriodendron Leaves
}

\author{
Fang-Fang Fu, ${ }_{1,2}$ Zhaodong Hao, ${ }^{1,2}$ Pengkai Wang, ${ }^{1,2}$ Ye Lu1,2 Liang-Jiao Xue, ${ }^{1,2}$ Guoyu Wei, ${ }^{3}$ Yanli Tian, ${ }^{4}$ Baishi Hu, ${ }^{4}$ \\ Haibin Xu, ${ }^{5}$ Jisen Shi, ${ }^{1,2}$ Tielong Cheng, ${ }^{5}$ Guibin Wang, ${ }^{2}$ Yin Yi, ${ }^{6,7}$ and Jinhui Chen ${ }^{1,2, \dagger}$ \\ ${ }^{1}$ Key Laboratory of Forest Genetics \& Biotechnology of Ministry of Education of China, Co-Innovation Center for Sustainable Forestry in \\ Southern China, Nanjing Forestry University, Nanjing, China \\ ${ }^{2}$ College of Forestry, Nanjing Forestry University, Nanjing, China \\ ${ }^{3}$ Shanghai Municipal Agricultural and Rural Affairs Commission, Shanghai, China \\ ${ }^{4}$ College of Plant Protection and Key Laboratory of Integrated Management of Crop Diseases and Pests, Ministry of Education, Nanjing \\ Agricultural University, Nanjing, China \\ ${ }^{5}$ College of Biology and the Environment, Nanjing Forestry University, Nanjing, China \\ ${ }^{6}$ State Forestry Administration Key Laboratory of Biodiversity Conservation in Karst Mountainous Areas of Southwestern China, Guizhou \\ Normal University, Guiyang, China \\ ${ }^{7}$ Guizhou Provincial Key Laboratory of Plant Physiology and Developmental Regulation, Guizhou Normal University, Guiyang, China \\ Accepted for publication 18 March 2020.
}

\begin{abstract}
Colletotrichum gloeosporioides is a hemibiotrophic pathogen causing significant losses to economically important crops and forest trees, including Liriodendron. To explore the interaction between $C$. gloeosporioides and Liriodendron and to identify the candidate genes determining the pathogenesis, we sequenced and assembled the whole genome of C. gloeosporioides Lc1 ( $\mathrm{CgLc} 1)$ using PacBio and Illumina next generation sequencing and performed a comparative genomic analysis between $\mathrm{CgLc} 1$ and $\mathrm{Cg} 01$, the latter being a described endophytic species of the $C$. gloeosporioides complex. Gene structure prediction identified 15,744 protein-coding genes and 837 noncoding RNAs. Species-specific genes were characterized using an ortholog analysis followed by a pathway enrichment analysis, which showed that genes specific to $\mathrm{CgLc} 1$ were enriched for the arginine biosynthetic process.
\end{abstract}

ABSTRACT
Furthermore, genome synteny analysis revealed that most of the protein-coding genes fell into collinear blocks. However, two clusters of polyketide synthase genes were identified to be specific for CgLc1, suggesting that they might have an important role in virulence control. Transcriptional regulators coexpressed with polyketide synthase genes were detected through a Weighted Correlation Network Analysis. Taken together, this work provides new insight into the virulence- and pathogenesis-associated genes present in C. gloeosporioides and its possible lifestyle.

Keywords: coexpression network analysis, Colletotrichum gloeosporioides, comparative genomic analysis, Liriodendron, polyketide synthase, whole-genome sequencing
The ascomycete genus Colletotrichum comprises about 600 species, which can infect $>3,200$ plant species, including many important crops and forest trees (Dean et al. 2012; O'Connell et al. 2012). Molecular phylogenetic analysis indicates that the genus Colletotrichum includes nine major clades and several small clusters together with isolated species (Cannon et al. 2012). The gloeosporioides clade is formed by $C$. gloeosporioides and another 21 closely related species based on the internal transcribed spacer (ITS) gene tree and morphological analysis (Weir et al. 2012). Sometimes, the species of this complex are designated as C. gloeosporioides because of their typical morphology. The

†Corresponding author: J. Chen; chenjh@njfu.edu.cn

Funding: This work was supported by the Joint Fund of the Natural Science Foundation of China and the Karst Science Research Center of Guizhou Province grant U1812401, Foundation of Jiangsu forestry bureau grant LYKJ[2017]42, Jiangsu Provincial Key Research and Development Program grant BE2017376, Qinglan Project of Jiangsu Province of China, the Nature Science Foundation of China grant 31770715, and Priority Academic Program Development of Jiangsu Higher Education Institutions.

F.-F. Fu and $\mathrm{Z}$. Hao contributed equally to this work.

*The $\boldsymbol{e}$-Xtra logo stands for "electronic extra" and indicates that one supplementary figure and six supplementary tables are published online.

The author(s) declare no conflict of interest.

(c) 2020 The American Phytopathological Society species of the C. gloeosporioides complex are hemibiotrophic pathogens that can infect $>470$ host plants, causing anthracnose at various developmental stages (Hyde et al. 2009). In some plants, especially medicinal plants, C. gloeosporioides is present as an endophyte (Kumar 2014). The different lifestyles of the C. gloeosporioides complex may be explained by genetic variation, host species, plant age, and abiotic environment (De Silva et al. 2017; Delaye et al. 2013).

C. gloeosporioides infection starts when its conidia contact the plant surface. Under favorable temperature and humidity conditions, conidia germinate to produce infection structures, such as an appressorium (Hiruma et al. 2010). Appressorial cells produce hydrolases to degrade the host cell wall to facilitate penetration and small-secreted proteins to manipulate the host cell (O'Connell et al. 2012). The pathogenicity of $C$. gloeosporioides is determined by many genes involved in the infection process. Through molecular cloning, many causal genes have been identified, including Colletotrichum hard surface-induced protein 1 gene, which acts during surface contact (Liu and Kolattukudy 1998); C. appressorium 20 during appressorium formation; nitrogen starvation-induced gene $C g D N 3$ during the biotrophic phase of primary infection (Stephenson et al. 2000); and CgCTR2 (copper transporter), which is involved in the regulation of the cellular copper balance for optimal germination (Barhoom et al. 2008). These genes may interact with each other and form regulatory gene networks, which tune the pathogenicity of $C$. gloeosporioides in different host plants under diverse environments. Transcriptional 
profiling using RNA sequencing revealed successive waves of gene expression during different stages of fungi infection (Gan et al. 2013; O'Connell et al. 2012). The study of the whole genome will provide a systematic view of key genes controlling fungal pathogenicity and provide clues for breeding of pathogenresistant plants.

In this study, we sequenced the whole genome of $C$. gloeosporioides Lc1, isolated from Liriodendron chinense leaves, and performed a comparative genomic analysis with the $\mathrm{Cg} 01$ isolate of C. gloeosporioides complex, originating from Huperzia serrata (Kang et al. 2019). The latter was reported as $C$. gloeosporioides undergoing an endophytic lifestyle. L. chinense together with the species Liriodendron tulipifera are desirable street, ornamental, and timber trees with distinctive leaves and attractive tulip-shaped flowers. The wood is soft, moderately light, and easily worked for furniture stock, veneer, and pulpwood. C. gloeosporioides has been reported to cause spot anthracnose disease to $L$. chinense (Choi et al. 2012), L. tulipifera (Lori et al. 2004), and the hybrid plants of $L$. chinense and L. tulipifera (Zhu et al. 2019), causing a significant economic loss. Our analysis indicated that gene clusters of polyketide synthase are expanded in the $\mathrm{CgLc} 1$ isolate compared with the $\mathrm{CgO} 1$ isolate, providing clues that help understand the specification of lifestyles in closely related species of the C. gloeosporioides complex.

\section{MATERIALS AND METHODS}

Isolation and identification of C. gloeosporioides. Anthracnose symptomatic leaves were collected from the tulip tree (L. chinense) on the Nanjing Forestry University (NFU) campus in May 2015. Infected leaf pieces were surface disinfected with $75 \%$ ethanol and rinsed in sterile deionized water; then, they were plated on potato dextrose agar (PDA) with addition of $100 \mu \mathrm{g} / \mathrm{ml}$ streptomycin and incubated at $25^{\circ} \mathrm{C}$ for $48 \mathrm{~h}$. Mycelia were then subcultured on new PDA plates to isolate and purify. Mycelial colonies were observed after 7 days, and conidia were inspected under a differential interference contrast (DIC) microscope (Imager D2; Zeiss). Fungal genomic DNA was extracted from mycelium on PDA plates using a DNA extraction kit (DP2031; Bioteke Co.). PCR was performed to amplify the full-length ITS sequence using the primer pair ITS1 (5'-TCCGTAGGTGAACCTGCGG-3') and ITS4 (5'-TCCTCCGCTTATTGATATGC-3') (White et al. 1990) to identify the species name of this isolation. The ITS sequence was deposited in NCBI GenBank with accession number MT065696.

Healthy young tulip tree leaves were collected and used for pathogenicity tests. After 10 days of culture, a sporulating C. gloeosporioides mycelia agar plug ( $5 \mathrm{~mm}$ in diameter) was cut from a plate and placed on wounded leaves to start infection. An agar plug from a sterile PDA plate was used as control. Both leaves were put into a petri dish with a moisture filter paper and inoculated in an incubator with a 12 -h photoperiod at $25^{\circ} \mathrm{C}$. Symptom development was observed on a daily basis.

Whole-genome sequencing and assembly. C. gloeosporioides Lc1 genomic DNA was sequenced using the Illumina Genome Analyzer X Ten and the PacBio Sequel System. DNA fragments of $250 \mathrm{bp}$ were used for Illumina library construction. Paired end reads of $100 \mathrm{bp}$ were obtained. Read quality was checked using FastQC V0.11.5 (Andrews 2010) and trimmed using Trimmomatic V0.36 (Bolger et al. 2014) followed by de novo assembling using Velvet version 1.2.10 (Zerbino and Birney 2008). A hybrid approach was applied to assemble the contigs into scaffolds using BLASR algorithms (Chaisson and Tesler 2012). The completeness of the genome assembly was evaluated using BUSCO v3.0.2 with Ascomycota data (Simão et al. 2015).

Genome annotation. MAKER was used to generate genome annotation, which integrates multiple tiers of coding evidence, such as ab initio gene predictions, transcript evidence, and protein evidence (Cantarel et al. 2008). SNAP (Korf 2004), GENEMARK (Lomsadze et al. 2005), and AUGUSTUS (Stanke et al. 2006) were used as ab initio gene predictors. Fungal transcripts from a ref_seq database and transcript/protein sequences of $C$. graminicola and C. higginsianum (O'Connell et al. 2012) were used as expression evidence. RepeatMasker was used to mask genome sequences (Smit et al. 1996), which were used as input for an MAKER pipeline. RNAmmer (Lagesen et al. 2007) and tRNAscan-SE (Lowe and Eddy 1997) were used to predict ribosomal RNAs (rRNAs) and transfer RNAs (tRNAs), respectively. Erpin (Lambert et al. 2004) was used to search for small nuclear RNA, small nucleolar RNA, and other noncoding RNAs.

For functional annotation, BLASTP was used to search against the SWISS-PROT and nonredundant (NR) protein databases of NCBI. The protein homology search in MycoCosm was used to assign the gene ontology (GO) terms, Kyoto Encyclopedia of Genes and Genomes (KEGG) pathways and eukaryotic clusters information to a certain protein (Grigoriev et al. 2013). For protein annotation, the eggNOG database (Muller et al. 2010) was searched using HMMER (Finn et al. 2011). Secreted proteins were predicted using SignalP (Bendtsen et al. 2004), and Carbohydrate-Active enZYmes were detected using CAZymes Analysis Toolkit (Park et al. 2010). The Pathogen-Host Interaction database (Urban et al. 2019) was used to predict potential pathogenically active proteins.

Clarification of Colletotrichum species from $\boldsymbol{H}$. serrata. An isolate of Colletotrichum species from $H$. serrata was reported as endophytic C. gloeosporioides (Kang et al. 2019). Before performing comparative genomic analysis, the sequences of barcoding genes were extracted from the genome sequence to double check the species name. These barcoding genes include actin (ACT), chitin synthase I (CHS-1), histone H3, and tubulin-2 (TUB2). The results from NCBI BLAST search indicated that the best hits of these genes are from $C$. kahawae rather than C. gloeosporioides (Supplementary Table S1). The genome sequence of $C$. kahawae $\mathrm{Cg} 01$ was annotated using the same pipeline as described above for $C$. gloeosporioides Lc1.

Molecular phylogeny analysis. The phylogenetic tree of C. gloeosporioides and other Colletotrichum species (Cannon et al. 2012) was constructed using a published approach (Gan et al. 2016). For each species, four gene sequences including ACT, CHS-1, ITS, and TUB2 were downloaded (Gan et al. 2016) or extracted from the genome sequence. The accession numbers of sequences and the names of species complex are shown in Supplementary Table S2. MAFFT (v7.215-e) and trimAL (v1.4) were used for alignment and trimming of the sequences, and RAxML ( $\mathrm{v}$ 8.2.4) was used for construction of a maximum likelihood species phylogeny using a GTRGAMMA model with 1,000 bootstrap replicates.

Orthologous gene analysis. In order to identify protein ortholog groups using OrthoFinder (Emms and Kelly 2015), protein sequences of 12 species in the genus Colletotrichum (including C. gloeosporioides $\mathrm{Lc1}$ and C. kahawae $\mathrm{Cg} 01$ ) and 17 other fungi species were downloaded from NCBI and Mycocosm (https:// mycocosm.jgi.doe.gov/mycocosm/home). BUSCO v3.0.2 (Simão et al. 2015) was used to assess the completeness of gene annotation using protein mode (Supplementary Table S3). An all-versus-all BLAST search was performed to identify gene pairs and screen for species-specific and clade-specific genes.

Synteny analysis and single-nucleotide polymorphism calling. Gene collinearity blocks between $C$. gloeosporioides Lc1 and C. kahawae Cg01 were detected using MCScanX (Wang et al. 2012). A Circos plot was further generated to visualize the collinearity blocks using the OmicCircos package $(\mathrm{Hu}$ et al. 2014). Scaffold sequences of C. gloeosporioides Lc1 and C. kahawae $\mathrm{Cg} 01$ were mapped to genome sequences of C. graminicola separately using MUMmer (Kurtz et al. 2004). The orders and orientations of scaffolds were determined based on the alignment results. The genes were placed onto the 
pseudochromosomes correspondingly. Gene blocks specific in C. gloeosporioides Lc1 were screened using an in-house python script through mining gaps between collinearity blocks. For single-nucleotide polymorphism (SNP) calling, the genome sequences of $C$. kahawae $\mathrm{Cg} 01$ were mapped onto the $C$. gloeosporioides Lc1 reference using MUMmer; SNPs were detected using the show-snps command of the MUMmer package.

TABLE 1. Genome assembly statistics of four Colletotrichum gloeosporioides strains

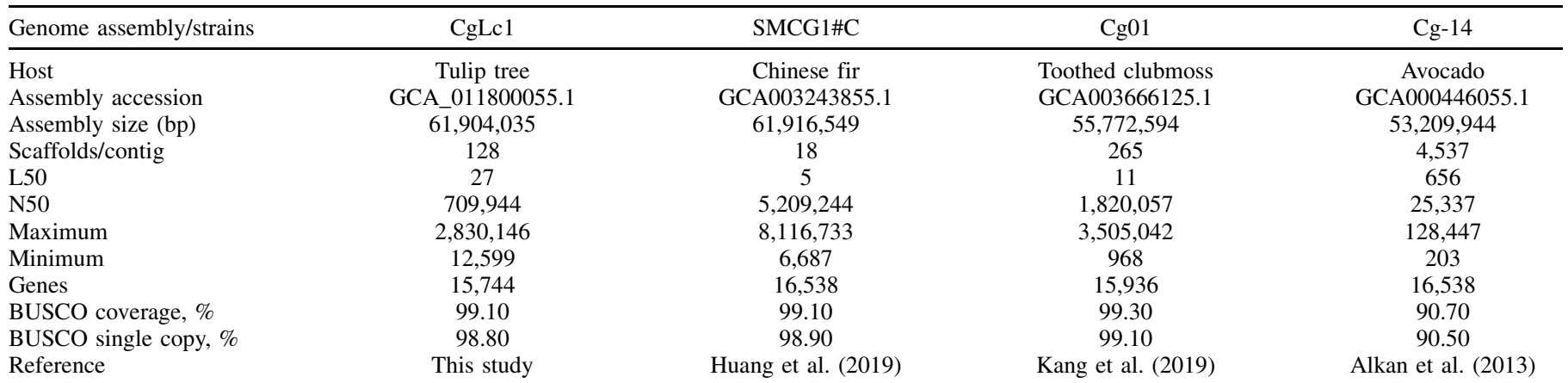
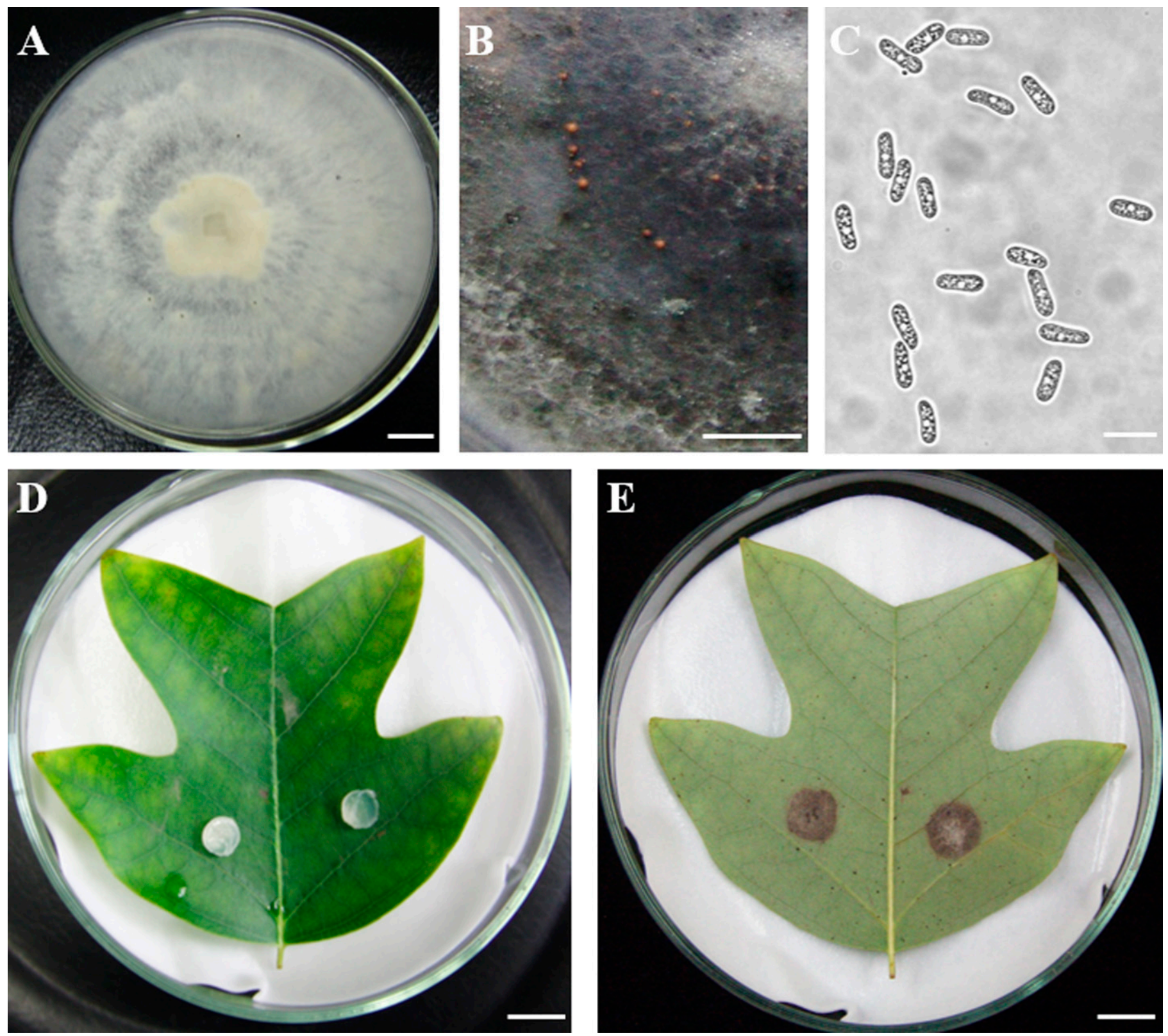

Fig. 1. Morphology and pathogenicity test of Colletotrichum gloeosporioides Lc1. A, Mycelial colony cultured on a potato dextrose agar plate. B, Orange conidia masses present on the plate after 10 days. C, Micrograph showing conidia. D and E, Anthracnose symptoms after wound-mediated inoculation with $\mathbf{D}$, control and E, C. gloeosporioides solution on Chinese tulip tree leaves after 6 days. Bars $=\mathbf{A}, \mathbf{B}, \mathbf{D}$, and $\mathbf{E}, 1 \mathrm{~cm} ; \mathbf{C}, 20 \mu \mathrm{m}$. 
Coexpression analysis. Gene expression data of C. gloeosporioides were downloaded from NCBI sequence read archive (SRA). The samples are described in Supplementary Table S4. Reads were trimmed using Trimmomatic v0.36 (Bolger et al. 2014) and mapped onto the CgLc1 reference using STAR v2.5.3a (Dobin et al. 2013). Read counts of each gene were calculated using featureCounts v1.6.2 tool (Liao et al. 2013), and gene expression levels were determined using DEseq2 (Love et al. 2014). A coexpression network was constructed using the Weighted Correlation Network Analysis (WGCNA) package (Langfelder and Horvath 2008). A GO enrichment test was performed using the topGO package (Alexa and Rahnenfuhrer 2010) and visualized using the pheatmap package (Kolde and Kolde 2015).

Data access. This Whole Genome Shotgun project has been deposited at DDBJ/ENA/GenBank under the accession WVTB00000000. The version described in this paper is version WVTB01000000.

\section{RESULTS}

Isolation and identification of $C$. gloeosporioides. Tulip tree leaves infected with spot anthracnose can be frequently found in the summer season. To identify which pathogen causes this

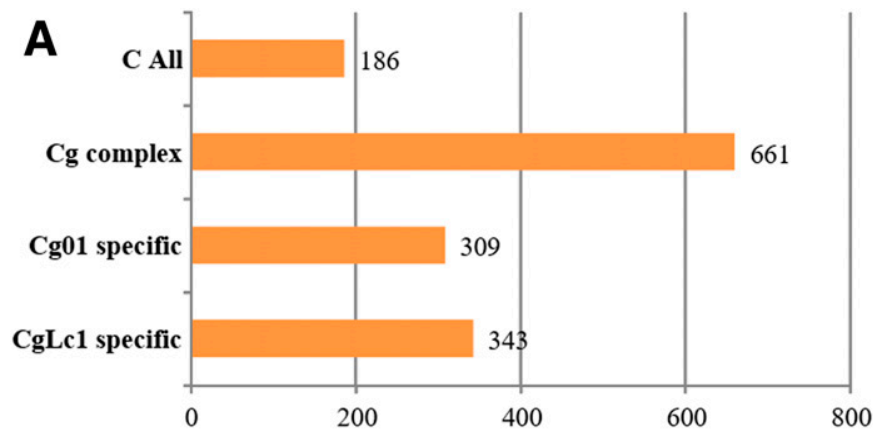

B

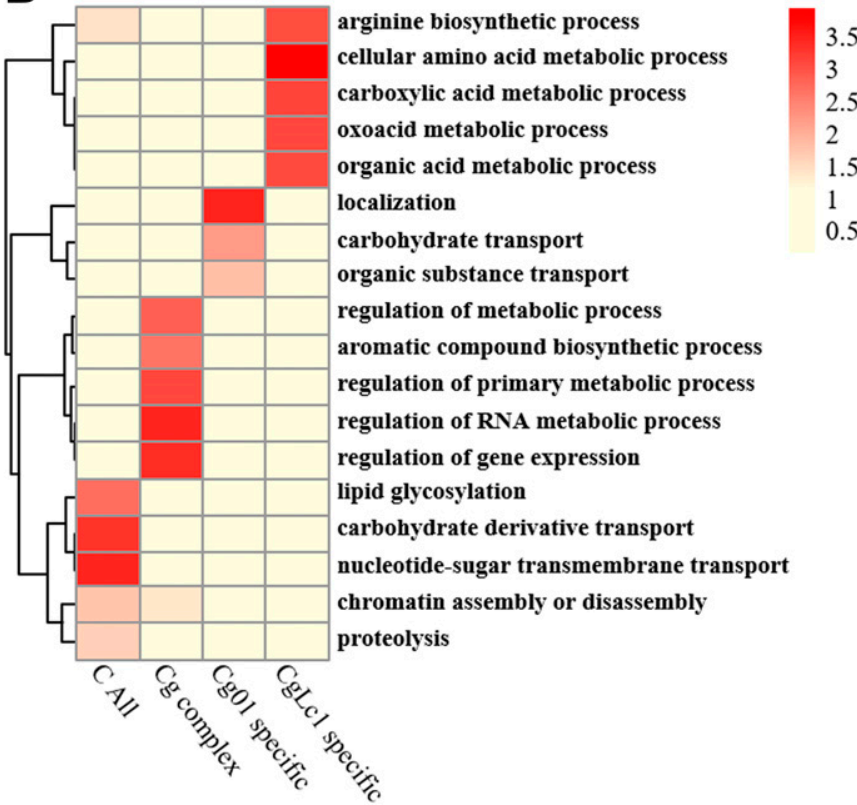

Fig. 2. Numbers and functions of genes in different Colletotrichum clades. A, Gene orthologs were identified using OrthoFinder. "C All" indicates common genes present in all Colletotrichum species used in our analysis. " $\mathrm{Cg}$ complex," "Cg01 specific," and "CgLc1 specific" indicate common genes in the $C$. gloeosporioides complex as well as specific genes in $\mathrm{CgO1}$ and $\mathrm{CgLc1}$. B, Gene ontology enrichment analysis performed on each gene list. $P$ values derived from the enrichment test by the topGO package are minus $\log _{10}$ transformed and visualized using a heat map plot. infection, diseased leaves (L. chinense) were collected on the NFU campus and incubated on PDA medium. ITS sequences (MT065696 in NCBI) amplified from the obtained fungi indicated the species as $C$. gloeosporioides $\mathrm{Lc1}(\mathrm{CgLc} 1)$. The fungi exhibited rounded colonies with smooth edges, starting off as white aerial hyphae at earlier stages and then slowly turning light gray (Fig. 1A). After 5 days of culture, tangerine-colored pionnotes were formed (Fig. 1B). Microscope examination indicates that the conidial cells were colorless, obtuse at both ends, and round or oblong, with two internal oil droplets (Fig. 1C). We inoculated the isolated fungal clone with healthy leaves to reproduce the disease symptoms and indeed, observed necrotic spots after 5 days of incubation on L. chinense leaves (Fig. 1D and E). These findings show that CgLc1 is the pathogen species causing anthracnose in Liriodendron leaves.

Genome assembly and annotation of CgLc1. To understand the genetic basis underlying the pathogenesis of $\mathrm{CgLc} 1$ and the involved genes, we sequenced the whole genome of C. gloeosporioides $\mathrm{CgLc1}$ and assembled it using a hybrid approach. We obtained $>3.29-\mathrm{Gb}(53 \times)$ PacBio reads and 11-Gb $(180 \times)$ Illumina paired end reads. De novo assemblies were first performed using Illumina reads, and then, PacBio reads were applied for scaffolding. The final assembled genome was $61.9 \mathrm{Mb}$ in total and consisted of 128 scaffolds in total, with the longest scaffold length being $2.8 \mathrm{Mb}$. The completeness of the assembly was then evaluated using the BUSCO database with the ascomycota dataset as a reference, resulting in a coverage rate of $99.1 \%$. The other assembly statistics were also comparable with previously reported isolates of $C$. gloeosporioides (Table 1). The genomic sequences of $A C T, C H S-1, I T S$, and TUB2 were extracted to confirm the species through phylogenetic analysis (Supplementary Fig. S1).

We used the MAKER pipeline to predict and annotate gene structures. In total, 15,744 protein-coding genes and 837 noncoding genes were identified. Among these protein-coding genes, 9,680 genes were annotated with potential functions using the SPROT database; 2,047 genes were annotated as encoding secreted proteins, 4,273 genes were predicted to play roles in pathogen-host interactions, and 2,344 genes were predicted to encode carbohydrate-active enzyme. Our genome sequence and gene annotation provide valuable resources for comparative analysis

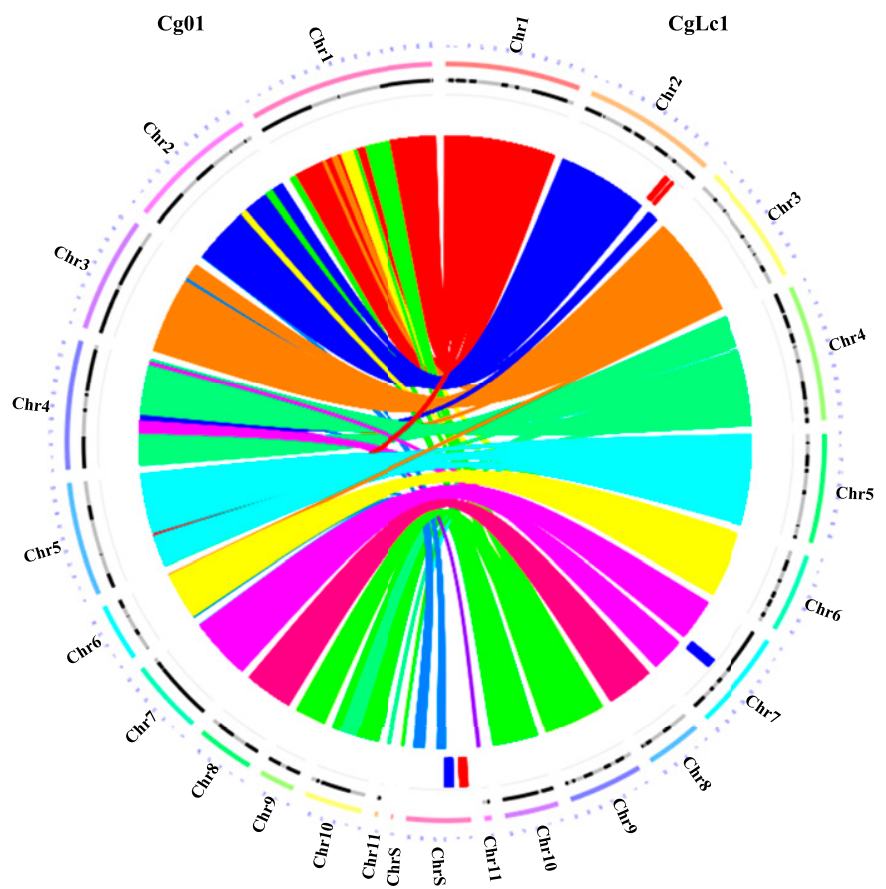

Fig. 3. Circos plot showing gene synteny between $\mathrm{Cg} 01$ (left) and CgLc1 (right). The scaffolds are organized into pseudochromosomes for visualization. Gene clusters specific for $\mathrm{CgLc1}$ are indicated by red and blue bars in the plot. 
of C. gloeosporioides to identify key genes underlying fungal pathogenicity.

Gene speciation of hemibiotrophic and endophytic isolates of $C$. gloeosporioides complex. Species of the $C$. gloeosporioides complex have a long history as pathogens. In contrast to the $\mathrm{CgLc} 1$ isolate from tulip tree leaves, isolate $\mathrm{Cg} 01$ from $H$. serrata was identified as an endophytic fungus (Kang et al. 2019). To clarify the species name of $\mathrm{Cg} 01$, the sequences of four barcoding genes were extracted from the genome sequence (in Materials and Methods) to search NCBI nucleotide databases. The results indicated that the isolate $\mathrm{Cg} 01$ is $C$. kahawae (Supplementary Table S1). During the process of species identification, species of the C. gloeosporioides complex were often defined as C. gloeosporioides in literature because they exhibit similar

A

Gene dendrogram and module colors
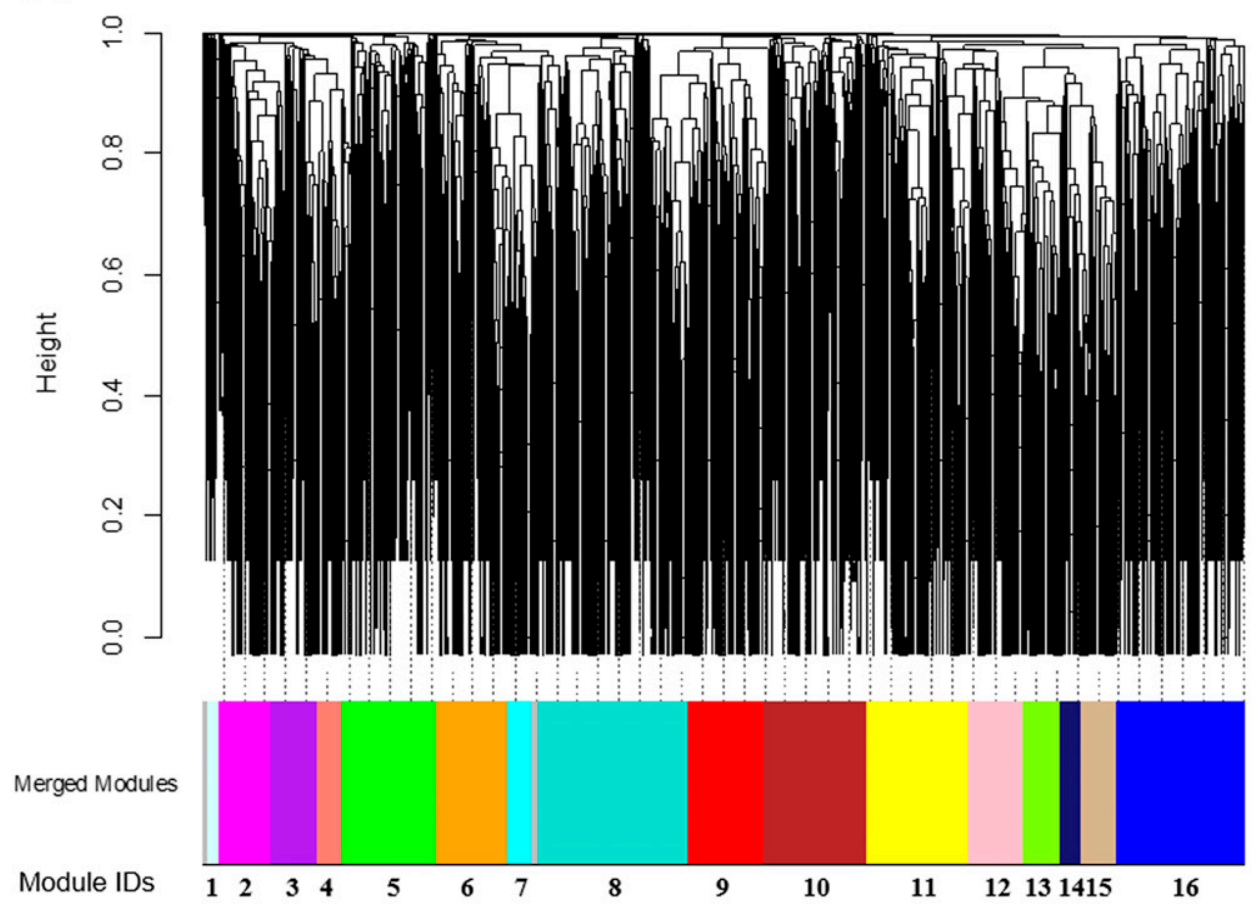

B
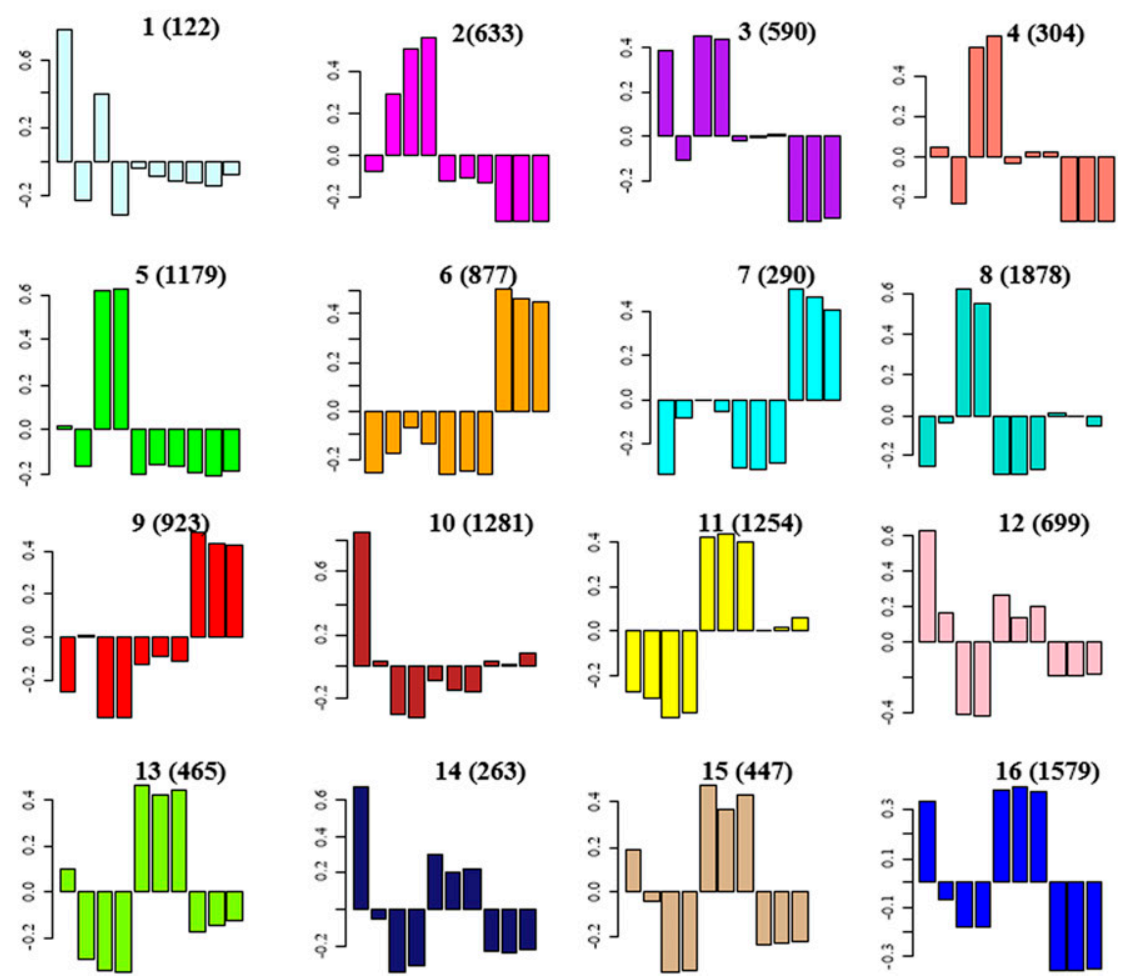

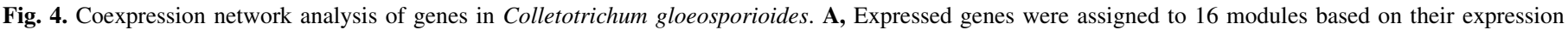

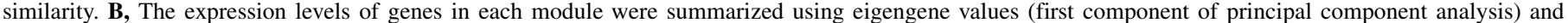
visualized using a bar plot. Different bars in each module represent the expression values from the 10 datasets used for the coexpression analysis. 
typical morphologies. In a 2016 publication, many endophytic species of the $C$. gloeosporioides complex found on the tea plant were named as $C$. gloeosporioides (Rabha et al. 2016). The species of the $C$. gloeosporioides complex undergoing different lifestyles provide valuable resources for comparative genomic analysis to explore the molecular mechanism determining the lifestyles.
To make sure the two genomes are comparable at the gene level, structural gene annotation was performed for isolate $\mathrm{Cg} 01$ using the same pipeline as $\mathrm{CgLc1} ; 15,936$ protein-coding genes were annotated in our analysis (Table 1). Genes were then assigned into ortholog groups using OrthoFinder software (Emms and Kelly 2015). The protein sequences of 27 fungal species were compared

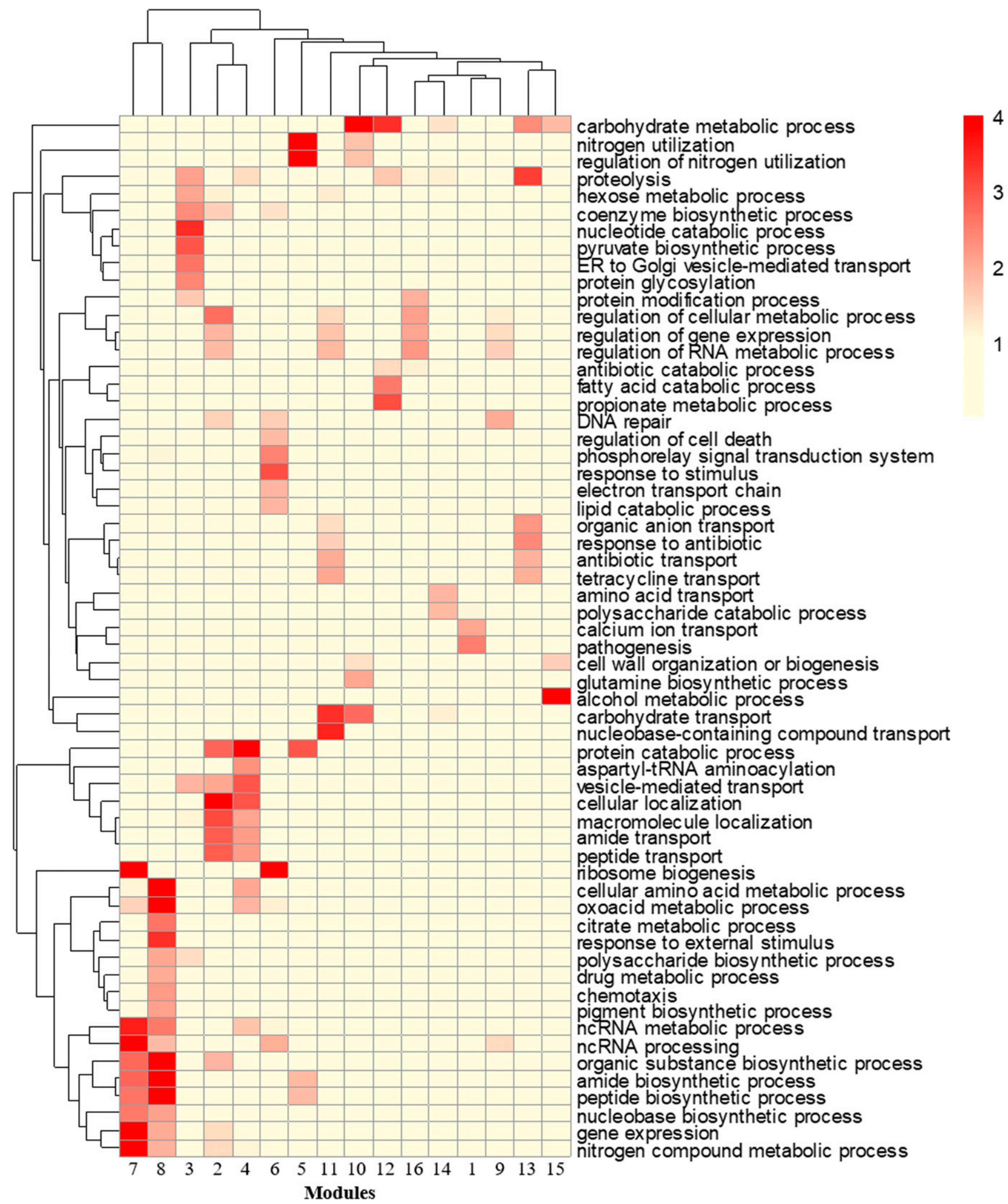

Fig. 5. Gene ontology enrichment of genes from different modules of the coexpression network. $P$ values were calculated using topGO and minus $\log _{10}$ transformed for visualization. ER, endoplasmic reticulum; tRNA, transfer RNA; and ncRNA, non-coding RNA. 
with two separate isolates of the $C$. gloeosporioides complex (Supplementary Table S3); 15,401 and 15,627 genes were assigned into 11,357 and 11,269 ortholog groups, respectively, and 343 and 309 genes were species specific in the isolates $\mathrm{CgLc1}$ and $\mathrm{Cg} 01$, respectively (Fig. 2A). In addition, 661 ortholog groups were present in the C. gloeosporioides complex, and 186 ortholog groups were shared by all 12 Colletotrichum species (Fig. 2A).

GO enrichment analysis reveals common functions of different gene sets. Genes specific in different clades (Fig. 2A) were subjected to $\mathrm{GO}$ enrichment analysis to compare their functional specificity (Fig. 2B). Genes involved in carbohydrate derivative transport and lipid glycosylation processes are enriched in genes specific to Colletotrichum species. Genes specific to the $C$. gloeosporioides complex are overrepresented with genes involved in the regulation of gene expression, the regulation of primary metabolic processes, aromatic compound biosynthetic processes, and organic substance transport processes. Comparing hemibiotrophic and endophytic isolates of the C. gloeosporioides complex, genes involved in arginine biosynthetic, carboxylic acid metabolic, and oxoacid metabolic processes are overrepresented in genes specific to the isolate $\mathrm{CgLc1}$, whereas genes involved in carbohydrate transport and organic substance transport were enriched in genes specific to the isolate $\mathrm{Cg} 01$. As reported previously, the mutations of genes in arginine biosynthesis resulted in severe reduction of the appressorial penetration and subsequent invasive growth in planta and in turn, reduced the pathogenicity of $C$. higginsianum (Takahara et al. 2012). Our results suggest that the increased number of genes involved in arginine biosynthesis in $C$. gloeosporioides $\mathrm{CgLc} 1$ may have caused the divergence of lifestyles between these two species of the C. gloeosporioides complex.

Genomic variation of hemibiotrophic and endophytic isolates. We analyzed the degree of genomic variation between the two isolates by determining the amount of SNPs/indels and presence/absence of synteny blocks. Most of the SNPs between these two isolates are located at intergenic and exon regions, whereas most of the indels are present in intergenic and intron regions (Supplementary Table S5). Synteny blocks within the isolate $\mathrm{CgLc1}$ and between isolates $\mathrm{CgLc1}$ and $\mathrm{Cg} 01$ were identified using MCscanX software (Wang et al. 2012). The results showed that most genes fell into synteny blocks, including 13,933 (88.5\%) CgLc1 genes and 13,927 (87.4\%) Cg01 genes (Fig. 3). Genes on four scaffolds of $\mathrm{CgLc1}$ have no orthologs in $\mathrm{Cg} 01$ (Fig. 3 ), of which most were also detected as $\mathrm{CgLc1} 1$-specific genes in the analysis outlined above for ortholog groups using OrthoFinder. The annotation from the NR and SPROT databases showed that most of these genes are proteins with unknown functions (Supplementary Table S6). The genes annotated using the SPROT database formed two major gene clusters, including five reducing polyketide synthase genes on scaffold consensus_39 (named as PKS_C39) and three genes encoding atrochrysone carboxylic acid synthase (named as PKS_C53), which is a nonreducing polyketide synthase (Awakawa et al. 2009), on scaffold consensus_53 (Supplementary Table S6). In addition, the $\arg -6$ gene, involved in arginine biosynthesis, as well as fusarin $\mathrm{C}$ synthetase, which also belongs to the polyketide synthase family (Hansen et al. 2012), are encoded in two CgLc1-specific blocks. Furthermore, previous reports have shown that polyketide synthase may function in melanin biosynthesis and determine virulence in Colletotrichum species (Fujii et al. 1999; Scindiya et al. 2019). Taken together, these findings suggest the important role of the polyketide synthase family in regulating the $C$. gloeosporioides species' lifestyle, which could be validated using gene deletion experiments.

Identification of the key regulators of polyketide synthase using coexpression network analysis. Diverse polyketides can be produced through the polyketide synthase pathway, such as toxins and melanins, which help fungi to get nutrition from dead plant tissues and protect themselves from oxidants and antimicrobial compounds (Nosanchuk and Casadevall 2003). To identify key regulators of polyketide biosynthesis pathways in $C$. gloeosporioides, we constructed a coexpression network using the publicly available datasets that include 10 RNAsequencing (RNA-seq) data from different C. gloeosporioides species (Supplementary Table S4). Sixteen network modules (Fig. 4A) were constructed using the WGCNA package (Langfelder and

TABLE 2. Transcription factors (TFs) coexpressed with polyketide synthase (PKS) genes

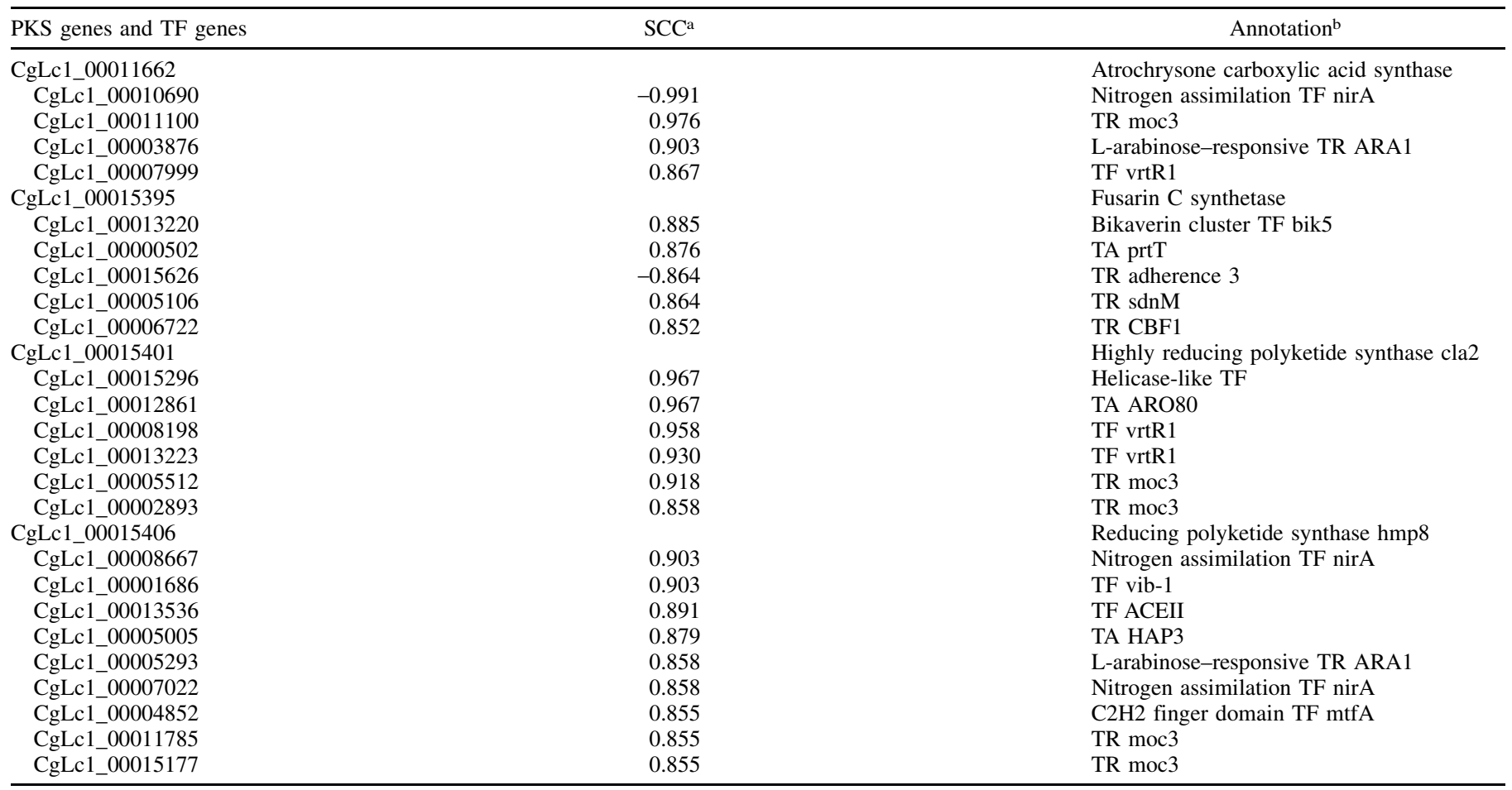

${ }^{a}$ SCC, Spearman correlation coefficient.

b TA, transcriptional activator; TR, transcription regulator. 
Horvath 2008). Genes in different modules exhibited distinct expression patterns. Genes that coexist in the same modules may undergo similar transcriptional regulation (Fig. 4B).

GO enrichment analysis was performed using genes in the 16 modules. As shown in Figure 5, genes in module 7 are enriched in biological processes, including nitrogen compound metabolism and peptide biosynthesis. The gene enrichment pattern of module 8 is similar to module 7; however, genes in pigment biosynthetic process were also overrepresented in this module. This analysis generated a valuable dataset to infer the regulatory functions of genes for $C$. gloeosporioides.

We mined the coexpression network to identify transcriptional regulators of metabolic genes in CgLc1-specific blocks (Table 2). Among these transcriptional regulators, the transcriptional activator spt 7 functions as a component of the histone acetylation complex, which is positively coexpressed with $C g L c 1 \_00011661$, an arginine biosynthesis gene. The nitrogen assimilation transcription factor nirA activates transcription of nitrate and nitrite reductases. One nirA gene is negatively correlated with a polyketide synthase (CgLc1_00011662), and another two nirA genes are positively correlated with a reducing polyketide synthase ( $\left.C g L c 1 \_00015406\right)$. These results provided putative regulators of polyketide synthase genes at the transcriptional level.

\section{DISCUSSION}

Hemibiotrophic and endophytic lifestyles of the $C$. gloeosporioides complex. Most Colletotrichum species are hemibiotrophic, whereas necrotrophic and endophytic species have also been observed (De Silva et al. 2017). A similar situation exists for the C. gloeosporioides species complex. A large number of cases has been reported where $C$. gloeosporioides species were found to be hemibiotrophic pathogens to crops, fruits, and forest trees (Weir et al. 2012). More and more species have been isolated that are endophytic to an increasing number of plants, especially medicinal plants (Kumar 2014). Species within the genetically closely related C. gloeosporioides complex or different isolates of a single species can exhibit different lifestyle patterns, providing a unique system for analyzing the underlying features that determine lifestyle, as well as a range of plant-pathogen interactions. In our analysis, hemibiotrophic $C$. gloeosporioides $\mathrm{Lc} 1(\mathrm{CgLc} 1)$ isolated from the tulip tree and endophytic $C$. kahawae $\mathrm{Cg} 01$ isolated from $H$. serrata were compared at the whole-genome level. We found that two clusters of virulence-promoting genes are specifically present in the hemibiotrophic $\mathrm{CgLc} 1$ isolate. This indicates that a single species may alter its lifestyle through gain or loss of numbers of genes or expansion or contraction of gene families. As in C. magna, mutation in a single locus can convert the pathogenic fungus into an endophytic mutualist (Freeman and Rodriguez 1993; Redman et al. 1999). Previous evolutionary analyses of fungal species have shown that a biotrophic lifestyle is evolutionarily stable among fungal species; however, endophytic and necrotrophic lifestyles can be easily switched in fungi at evolutionary and even ecological timescales (Delaye et al. 2013). Lifestyles switching can even be achieved by horizontal gene transfer, which has been reported to affect the host range of fungi (Mehrabi et al. 2011).

Polyketide synthase genes in CgLc1-specific clusters and its regulators. We detected three and six polyketide synthase genes in the two CgLc1-specific gene clusters (PKS_C39 and PKS_ C53, respectively), which catalyze the biosynthesis of polyketide from acyl-coenzyme A precursors (Supplementary Table S6). Polyketides are a large family of compounds naturally produced by bacteria, fungi, and plants, including clinically important drugs such as tetracycline, daunorubicin, and rapamycin (Shen 2003). During the interaction between necrotrophic fungi and their hosts or at the necrotrophic infection stage of hemibiotrophic fungi, polyketides are produced as toxins to kill plant cells for nutrition acquisition. Cercospora spp. produce perylenequinone polyketide toxin, which can be activated by light and produce reactive oxygen species to trigger cell death (Choquer et al. 2005). Dothistroma septosporum produces the polyketide dothistromin, which is important for pathogenicity and sporulation (Kabir et al. 2015). Melanins are another group of polyketide closely related with pathogenicity of fungi (Nosanchuk and Casadevall 2003). Melanization is important in microbial pathogenesis because it has been associated with virulence in a high number of microorganisms. Melanin in the cell wall forms a physical barrier between the environment and the cell membrane and contributes to virulence by reducing the susceptibility of melanized fungi to host defense mechanisms (Nosanchuk and Casadevall 2003).

A coexpression network was constructed to identify regulatory genes of polyketide synthase and other metabolic genes. This resource provides a dataset to mine interacting gene pairs in C. gloeosporioides. Several transcription factors were identified that coexpressed with the polyketide synthase genes, including $M t f A$, which has been reported to be a master transcription factor governing polyketide production (Zhuang et al. 2016). Three transcription factors controlling the nitrogen and carbon metabolism were also identified, including nirA, a pathway-specific regulatory gene of nitrate assimilation (Burger et al. 1991); ARO80, a positive feedback regulator of aromatic alcohol production, which depends on the availability of nitrogen (Chen and Fink 2006); and vib-1, a major transcriptional regulator of the nitrogen and carbon starvation response, which promotes the production of extracellular proteases under resource-limited conditions (Dementhon et al. 2006). Moc3, a transcription factor involved in stress response, was also identified by its coexpression with several polyketide synthase genes (Goldar et al. 2005). These results indicate the fine regulation of polyketide biosynthesis in $C$. gloeosporioides under diverse conditions during the interaction with plant hosts.

The identification of polyketide synthase genes and their transcriptional regulators will provide more clues for pathogen control. Knowing which genes cause pathogenesis will facilitate the identification of particular toxins and allow for the introduction of enzymes degrading specific toxins into host plants, such as the genus Liriodendron, to increase their resistance to C. gloeosporioides. Furthermore, secondary metabolic pathways can also be engineered to produce metabolites interacting with $C$. gloeosporioides transcription factors. Finally, our analysis also provides valuable candidate genes to test how certain lifestyles were specified within the $C$. gloeosporioides species complex. Previous analysis of the endophytic microbe genomes has revealed genes involved in antibiotic resistance, antibiotic production, endophytic secretory system, and carbohydrate active enzymes as determining factors of the bioactivities and colonizing preferences (Kaul et al. 2016; Knapp et al. 2018).

\section{LITERATURE CITED}

Alexa, A., and Rahnenfuhrer, J. 2010. topGO: Enrichment Analysis for Gene Ontology. R Package Version 2:2010.

Alkan, N., Meng, X., Friedlander, G., Reuveni, E., Sukno, S., Sherman, A., Thon, M., Fluhr, R., and Prusky, D. 2013. Global aspects of pacC regulation of pathogenicity genes in Colletotrichum gloeosporioides as revealed by transcriptome analysis. Mol. Plant-Microbe Interact. 26:1345-1358.

Andrews, S. 2010. FastQC: A Quality Control Tool for High Throughput Sequence Data. Babraham Bioinformatics. Babraham Institute, Cambridge, United Kingdom.

Awakawa, T., Yokota, K., Funa, N., Doi, F., Mori, N., Watanabe, H., and Horinouchi, S. 2009. Physically discrete $\beta$-lactamase-type thioesterase catalyzes product release in atrochrysone synthesis by iterative type I polyketide synthase. Chem. Biol. 16:613-623.

Barhoom, S., Kupiec, M., Zhao, X., Xu, J. R., and Sharon, A. 2008. Functional characterization of $\mathrm{CgCTR} 2$, a putative vacuole copper transporter that is involved in germination and pathogenicity in Colletotrichum gloeosporioides. Eukaryot. Cell 7:1098-1108.

Bendtsen, J. D., Nielsen, H., von Heijne, G., and Brunak, S. 2004. Improved prediction of signal peptides: SignalP 3.0. J. Mol. Biol. 340:783-795.

Bolger, A. M., Lohse, M., and Usadel, B. 2014. Trimmomatic: A flexible trimmer for Illumina sequence data. Bioinformatics 30:2114-2120. 
Burger, G., Strauss, J., Scazzocchio, C., and Lang, B. F. 1991. nirA, the pathway-specific regulatory gene of nitrate assimilation in Aspergillus nidulans, encodes a putative GAL4-type zinc finger protein and contains four introns in highly conserved regions. Mol. Cell. Biol. 11:5746-5755.

Cannon, P. F., Damm, U., Johnston, P. R., and Weir, B. S. 2012. Colletotrichum - current status and future directions. Stud. Mycol. 73:181-213.

Cantarel, B. L., Korf, I., Robb, S. M., Parra, G., Ross, E., Moore, B., Holt, C., Alvarado, A. S., and Yandell, M. 2008. MAKER: An easy-to-use annotation pipeline designed for emerging model organism genomes. Genome Res. 18: 188-196.

Chaisson, M. J., and Tesler, G. 2012. Mapping single molecule sequencing reads using basic local alignment with successive refinement (BLASR): Application and theory. BMC Bioinformatics 13:238.

Chen, H., and Fink, G. R. 2006. Feedback control of morphogenesis in fungi by aromatic alcohols. Genes Dev. 20:1150-1161.

Choi, O., Choi, O., Kwak, Y. S., Kim, J., and Kwon, J. H. 2012. Spot anthracnose disease caused by Colletotrichum gloeosporioides on tulip tree in Korea. Mycobiology 40:82-84.

Choquer, M., Dekkers, K. L., Chen, H.-Q., Cao, L., Ueng, P. P., Daub, M. E., and Chung, K.-R. 2005. The CTB1 gene encoding a fungal polyketide synthase is required for cercosporin biosynthesis and fungal virulence of Cercospora nicotianae. Mol. Plant-Microbe Interact. 18:468-476.

De Silva, D. D., Crous, P. W., Ades, P. K., Hyde, K. D., and Taylor, P. W. J. 2017. Life styles of Colletotrichum species and implications for plant biosecurity. Fungal Biol. Rev. 31:155-168.

Dean, R., Van Kan, J. A. L., Pretorius, Z. A., Hammond-Kosack, K. E., Di Pietro, A., Spanu, P. D., Rudd, J. J., Dickman, M., Kahmann, R., Ellis, J., and Foster, G. D. 2012. The top 10 fungal pathogens in molecular plant pathology. Mol. Plant Pathol. 13:414-430.

Delaye, L., García-Guzmán, G., and Heil, M. 2013. Endophytes versus biotrophic and necrotrophic pathogens-are fungal lifestyles evolutionarily stable traits? Fungal Divers. 60:125-135.

Dementhon, K., Iyer, G., and Glass, N. L. 2006. VIB-1 is required for expression of genes necessary for programmed cell death in Neurospora crassa. Eukaryot. Cell 5:2161-2173.

Dobin, A., Davis, C. A., Schlesinger, F., Drenkow, J., Zaleski, C., Jha, S., Batut, P., Chaisson, M., and Gingeras, T. R. 2013. STAR: Ultrafast universal RNA-seq aligner. Bioinformatics 29:15-21.

Emms, D. M., and Kelly, S. 2015. OrthoFinder: Solving fundamental biases in whole genome comparisons dramatically improves orthogroup inference accuracy. Genome Biol. 16:157.

Finn, R. D., Clements, J., and Eddy, S. R. 2011. HMMER web server: Interactive sequence similarity searching. Nucleic Acids Res. 39(suppl 2): W29-W37.

Freeman, S., and Rodriguez, R. J. 1993. Genetic conversion of a fungal plant pathogen to a nonpathogenic, endophytic mutualist. Science 260:75-78

Fujii, I., Mori, Y., Watanabe, A., Kubo, Y., Tsuji, G., and Ebizuka, Y. 1999. Heterologous expression and product identification of Colletotrichum lagenarium polyketide synthase encoded by the PKS1 gene involved in melanin biosynthesis. Biosci. Biotechnol. Biochem. 63:1445-1452.

Gan, P., Ikeda, K., Irieda, H., Narusaka, M., O'Connell, R. J., Narusaka, Y., Takano, Y., Kubo, Y., and Shirasu, K. 2013. Comparative genomic and transcriptomic analyses reveal the hemibiotrophic stage shift of Colletotrichum fungi. New Phytol. 197:1236-1249.

Gan, P., Narusaka, M., Kumakura, N., Tsushima, A., Takano, Y., Narusaka, Y., and Shirasu, K. 2016. Genus-wide comparative genome analyses of Colletotrichum species reveal specific gene family losses and gains during adaptation to specific infection lifestyles. Genome Biol. Evol. 8:1467-1481.

Goldar, M. M., Jeong, H. T., Tanaka, K., Matsuda, H., and Kawamukai, M. 2005. Moc3, a novel Zn finger type protein involved in sexual development, ascus formation, and stress response of Schizosaccharomyces pombe. Curr. Genet. 48:345-355.

Grigoriev, I. V., Nikitin, R., Haridas, S., Kuo, A., Ohm, R., Otillar, R., Riley, R., Salamov, A., Zhao, X., and Korzeniewski, F. 2013. MycoCosm portal: Gearing up for 1000 fungal genomes. Nucleic Acids Res. 42: D699-D704.

Hansen, F. T., Sørensen, J. L., Giese, H., Sondergaard, T. E., and Frandsen, R. J. N. 2012. Quick guide to polyketide synthase and nonribosomal synthetase genes in Fusarium. Int. J. Food Microbiol. 155:128-136.

Hiruma, K., Onozawa-Komori, M., Takahashi, F., Asakura, M., Bednarek, P., Okuno, T., Schulze-Lefert, P., and Takano, Y. 2010. Entry mode-dependent function of an indole glucosinolate pathway in Arabidopsis for nonhost resistance against anthracnose pathogens. Plant Cell 22:2429-2443.

Hu, Y., Yan, C., Hsu, C.-H., Chen, Q.-R., Niu, K., Komatsoulis, G. A., and Meerzaman, D. 2014. OmicCircos: A simple-to-use R package for the circular visualization of multidimensional omics data. Cancer Inform. 13: 13-20.

Huang, L., Kim, K.-T., Yang, J.-Y., Song, H., Choi, G., Jeon, J., Cheong, K., Ko, J., Xu, H., and Lee, Y.-H. 2019. A high-quality draft genome sequence of Colletotrichum gloeosporioides sensu stricto SMCG1\# C, a causal agent of anthracnose on Cunninghamia lanceolata in China. Mol. Plant-Microbe Interact. 32:139-141.

Hyde, K. D., Cai, L., Cannon, P. F., Crouch, J. A., Crous, P. W., Damm, U., Goodwin, P. H., Chen, H., Johnston, P. R., Jones, E. B. G., Liu, Z. Y., McKenzie, E. H. C., Moriwaki, J., Noireung, P., Pennycook, S. R., Pfenning, L. H., Prihastuti, H., Sato, T., Shivas, R. G., Tan, Y. P., Taylor, P. W. J., Weir, B. S., Yang, Y. L., and Zhang, J. Z. 2009. Colletotrichum-Names in current use. Fungal Divers. 39:147-182.

Kabir, M., Ganley, R., and Bradshaw, R. 2015. Dothistromin toxin is a virulence factor in dothistroma needle blight of pines. Plant Pathol. 64:225-234.

Kang, X., Liu, C., Shen, P., Hu, L., Lin, R., Ling, J., Xiong, X., Xie, B., and Liu, D. 2019. Genomic characterization provides new insights into the biosynthesis of the secondary metabolite huperzine $\mathrm{a}$ in the endophyte Colletotrichum gloeosporioides Cg01. Front. Microbiol. 9:3237.

Kaul, S., Sharma, T., and Dhar, M. K. 2016. "Omics" tools for better understanding the plant-endophyte interactions. Front. Plant Sci. 7:955.

Knapp, D. G., Németh, J. B., Barry, K., Hainaut, M., Henrissat, B., Johnson, J., Kuo, A., Lim, J. H. P., Lipzen, A., Nolan, M., Ohm, R. A., Tamás, L., Grigoriev, I. V., Spatafora, J. W., Nagy, L. G., and Kovács, G. M. 2018. Comparative genomics provides insights into the lifestyle and reveals functional heterogeneity of dark septate endophytic fungi. Sci. Rep. 8:6321.

Kolde, R., and Kolde, M. R. 2015. pheatmap: Pretty Heatmaps. R package Version 1.0.12.

Korf, I. 2004. Gene finding in novel genomes. BMC Bioinformatics 5:59.

Kumar, G. A. 2014. Colletotrichum gloeosporioides: Biology, pathogenicity and management in India. J. Plant Physiol. Pathol. 2:2.

Kurtz, S., Phillippy, A., Delcher, A. L., Smoot, M., Shumway, M., Antonescu, C., and Salzberg, S. L. 2004. Versatile and open software for comparing large genomes. Genome Biol. 5:R12.

Lagesen, K., Hallin, P., Rødland, E. A., Stærfeldt, H.-H., Rognes, T., and Ussery, D. W. 2007. RNAmmer: Consistent and rapid annotation of ribosomal RNA genes. Nucleic Acids Res. 35:3100-3108.

Lambert, A., Fontaine, J.-F., Legendre, M., Leclerc, F., Permal, E., Major, F., Putzer, H., Delfour, O., Michot, B., and Gautheret, D. 2004. The ERPIN server: An interface to profile-based RNA motif identification. Nucleic Acids Res. 32(suppl 2):W160-W165.

Langfelder, P., and Horvath, S. 2008. WGCNA: An R package for weighted correlation network analysis. BMC Bioinformatics 9:559.

Liao, Y., Smyth, G. K., and Shi, W. 2013. featureCounts: An efficient general purpose program for assigning sequence reads to genomic features. Bioinformatics 30:923-930.

Liu, Z. M., and Kolattukudy, P. E. 1998. Identification of a gene product induced by hard-surface contact of Colletotrichum gloeosporioides conidia as a ubiquitin-conjugating enzyme by yeast complementation. J. Bacteriol. 180:3592-3597.

Lomsadze, A., Ter-Hovhannisyan, V., Chernoff, Y. O., and Borodovsky, M. 2005. Gene identification in novel eukaryotic genomes by self-training algorithm. Nucleic Acids Res. 33:6494-6506.

Lori, G. A., Alippi, A. M., and Dimenna, S. 2004. First report of species of Colletotrichum causing leaf blotch of Liriodendron tulipifera in Argentina. Plant Dis. 88:1381.

Love, M. I., Huber, W., and Anders, S. 2014. Moderated estimation of fold change and dispersion for RNA-seq data with DESeq2. Genome Biol. 15:550.

Lowe, T. M., and Eddy, S. R. 1997. tRNAscan-SE: A program for improved detection of transfer RNA genes in genomic sequence. Nucleic Acids Res. 25:0955-0964

Mehrabi, R., Bahkali, A. H., Abd-Elsalam, K. A., Moslem, M., Ben M'Barek, S., Gohari, A. M., Jashni, M. K., Stergiopoulos, I., Kema, G. H. J., and de Wit, P. 2011. Horizontal gene and chromosome transfer in plant pathogenic fungi affecting host range. FEMS Microbiol. Rev. 35:542-554.

Muller, J., Szklarczyk, D., Julien, P., Letunic, I., Roth, A., Kuhn, M., Powell, S., von Mering, C., Doerks, T., and Jensen, L. J. 2010. eggNOG v2. 0: Extending the evolutionary genealogy of genes with enhanced nonsupervised orthologous groups, species and functional annotations. Nucleic Acids Res. 38(suppl 1):D190-D195.

Nosanchuk, J. D., and Casadevall, A. 2003. The contribution of melanin to microbial pathogenesis. Cell. Microbiol. 5:203-223.

O’Connell, R. J., Thon, M. R., Hacquard, S., Amyotte, S. G., Kleemann, J., Torres, M. F., Damm, U., Buiate, E. A., Epstein, L., Alkan, N., Altmuller, J., Alvarado-Balderrama, L., Bauser, C. A., Becker, C., Birren, B. W., Chen, Z., Choi, J., Crouch, J. A., Duvick, J. P., Farman, M. A., Gan, P., Heiman, D., Henrissat, B., Howard, R. J., Kabbage, M., Koch, C., Kracher, B., Kubo, Y., Law, A. D., Lebrun, M. H., Lee, Y. H., Miyara, I., Moore, N., Neumann, U., Nordstrom, K., Panaccione, D. G., Panstruga, R., Place, M., Proctor, R. H., Prusky, D., Rech, G., Reinhardt, R., Rollins, J. A., Rounsley, S., Schardl, C. L., Schwartz, D. C., Shenoy, N., Shirasu, K., Sikhakolli, U. R., Stuber, K., Sukno, S. A., Sweigard, J. A., Takano, Y., Takahara, H., Trail, F., van der Does, H. C., Voll, L. M., Will, I., Young, S., Zeng, Q., Zhang, J., 
Zhou, S., Dickman, M. B., Schulze-Lefert, P., Ver Loren van Themaat, E., Ma, L. J., and Vaillancourt, L. J. 2012. Lifestyle transitions in plant pathogenic Colletotrichum fungi deciphered by genome and transcriptome analyses. Nat. Genet. 44:1060-1065.

Park, B. H., Karpinets, T. V., Syed, M. H., Leuze, M. R., and Uberbacher, E. C. 2010. CAZymes Analysis Toolkit (CAT): Web service for searching and analyzing carbohydrate-active enzymes in a newly sequenced organism using CAZy database. Glycobiology 20:1574-1584.

Rabha, A. J., Naglot, A., Sharma, G. D., Gogoi, H. K., Gupta, V. K., Shreemali, D. D., and Veer, V. 2016. Morphological and molecular diversity of endophytic Colletotrichum gloeosporioides from tea plant, Camellia sinensis (L.) O. Kuntze of Assam, India. J. Genet. Eng. Biotechnol. 14:181-187.

Redman, R. S., Ranson, J. C., and Rodriguez, R. J. 1999. Conversion of the pathogenic fungus Colletotrichum magna to a nonpathogenic, endophytic mutualist by gene disruption. Mol. Plant-Microbe Interact. 12:969-975.

Scindiya, M., Malathi, P., Kaverinathan, K., Sundar, A. R., and Viswanathan, R. 2019. RNA-mediated silencing of PKS1 gene in Colletotrichum falcatum causing red rot in sugarcane. Eur. J. Plant Pathol. 153:371-384.

Shen, B. 2003. Polyketide biosynthesis beyond the type I, II and III polyketide synthase paradigms. Curr. Opin. Chem. Biol. 7:285-295.

Simão, F. A., Waterhouse, R. M., Ioannidis, P., Kriventseva, E. V., and Zdobnov, E. M. 2015. BUSCO: Assessing genome assembly and annotation completeness with single-copy orthologs. Bioinformatics 31:3210-3212.

Smit, A. F., Hubley, R., and Green, P. 1996. RepeatMasker Open-3.0.

Stanke, M., Schöffmann, O., Morgenstern, B., and Waack, S. 2006. Gene prediction in eukaryotes with a generalized hidden Markov model that uses hints from external sources. BMC Bioinformatics 7:62.

Stephenson, S.-A., Hatfield, J., Rusu, A. G., Maclean, D. J., and Manners, J. M. 2000. CgDN3: An essential pathogenicity gene of Colletotrichum gloeosporioides necessary to avert a hypersensitive-like response in the host Stylosanthes guianensis. Mol. Plant-Microbe Interact. 13:929-941.

Takahara, H., Huser, A., and O'Connell, R. 2012. Two arginine biosynthesis genes are essential for pathogenicity of Colletotrichum higginsianum on Arabidopsis. Mycology 3:54-64.

Urban, M., Cuzick, A., Seager, J., Wood, V., Rutherford, K., Venkatesh, S. Y., De Silva, N., Martinez, M. C., Pedro, H., Yates, A. D., Hassani-Pak, K., and Hammond-Kosack, K. E. 2019. PHI-base: The pathogen-host interactions database. Nucleic Acids Res. 48:D613-D620.

Wang, Y., Tang, H., Debarry, J. D., Tan, X., Li, J., Wang, X., Lee, T., Jin, H., Marler, B., Guo, H., Kissinger, J. C., and Paterson, A. H. 2012. MCScanX: A toolkit for detection and evolutionary analysis of gene synteny and collinearity. Nucleic Acids Res. 40:e49.

Weir, B. S., Johnston, P. R., and Damm, U. 2012. The Colletotrichum gloeosporioides species complex. Stud. Mycol. 73:115-180.

White, T. J., Bruns, T., Lee, S., and Taylor, J. 1990. Amplification and direct sequencing of fungal ribosomal RNA genes for phylogenetics. Pages 315-322 in: PCR Protocols: A Guide to Methods and Applications. M. A. Innis, D. H. Gelfand, J. J. Sninsky, and T. J. White, eds. Academic Press, San Diego, CA.

Zerbino, D. R., and Birney, E. 2008. Velvet: Algorithms for de novo short read assembly using de Bruijn graphs. Genome Res. 18:821-829.

Zhu, L. H., Wan, Y., Zhu, Y. N., Huang, L., Liu, C. L., and Li, D. W. 2019. First report of species of Colletotrichum causing leaf spot of Liriodendron chinense $\times$ tulipifera in China. Plant Dis. 103:1431-1432.

Zhuang, Z., Lohmar, J. M., Satterlee, T., Cary, J. W., and Calvo, A. M. 2016. The master transcription factor mtfA governs aflatoxin production, morphological development and pathogenicity in the fungus Aspergillus flavus. Toxins (Basel) 8:29. 